\title{
Hydrops fetalis secondary to supradiaphragmatic extrapulmonary sequestration with congenital cystic adenomatoid malformation Shukla $\mathrm{S}^{1}$, Kini $\mathrm{H}^{2}$, Ilias $\mathrm{ML}^{3}$, Gautam $\mathrm{K}^{4}$ \\ ${ }^{1}$ Department of Pathology, Dr. Ram Manohar Lohia Institute of Medical Sciences, Lucknow, Uttar Pradesh, India ${ }^{2}$ Department of Pathology, Kasturba Medical College, Mangalore, Karnataka, India. ${ }^{3}$ Department of Pathology, M.E.S Medical College, Perinthalmanna, Kerala, Indiq. \\ ${ }^{4}$ Department of Pathology, Samyak Diagnostic Pvt. Ltd, Lalitpur, Nepal.
}

\section{Keywords:}

Adenomatoid malformation;

Cystic;

Extralobar sequestration;

Pulmonary sequestration

\begin{abstract}
Congenital cystic adenomatoid malformation (CCAM) is a hamartomatous lesion. A 30 year old woman, G2P1L1, in the 29th week of gestation presented with pain abdomen, chest pain, cough with expectoration, fever and inability to appreciate fetal movements of 2 days duration. Clinically, she had pneumonia. An ultrasound revealed a single fetus in breech presentation with features of hydrops fetalis, hypoplastic left lung, mediastinal shift to the left and poor diastolic and systolic flow in the umbilical artery.

A still born male baby delivered subsequently was found at autopsy to have hydrops fetalis, supradiaphragmatic right sided extralobar sequestration (ELS) with associated congenital cystic adenomatoid malformation (CCAM). The right lung also showed CCAM. There were no other associated anomalies. We present a rare case of ELS with CCAM.
\end{abstract}

\section{INTRODUCTION}

Congenital cystic adenomatoid malformation (CCAM) is a hamartomatous lesion characterized by abnormal bronchial and bronchiolar-like structures of varying sizes and distribution. It is associated with $34.4 \%$ stillbirths. Nonimmune hydrops fetalis has been described in association with numerous conditions of the fetus in-utero. ${ }^{1}$ Thoracic pathology commonly associated with hydrops include congenital cystic adenomatoid malformation of the lung

\footnotetext{
Correspondence:

Dr. Saumya Shukla, MBBS, MD

Assistant Professor, Dept of Pathology

Dr Ram Manohar Lohia Institute of Medical Sciences,

Lucknow-India

Email:saumyavarun@gmail.com
}

(CCAM) (30\%), Chondrodysplasia (25\%), right sided diaphragmatic hernia $(15 \%)$, pulmonary sequestration (8\%), etc.1 Extra-lobar pulmonary sequestration (ELS) is defined as the presence of a mass of abnormal pulmonary tissue outside the visceral pleura, that does not communicate with the tracheobronchial tree and is supplied by an anomalous systemic artery. Congenital cystic adenomatoid malformation is a hamartomatous lesion characterized by the presence of abnormal bronchial and bronchiolar like structures of varying sizes and distribution. In a series of 15 cases reviewed by Stocker and Kagan-Hallet, one case contained CCAM with extra-lobar pulmonary sequestration. ${ }^{2}$ Subsequently, Zangwill and Stocker described a case of CCAM with an ELS and stated that association occurred 


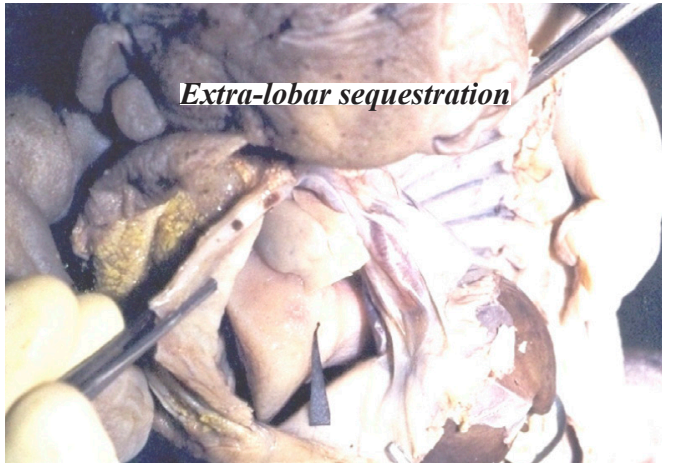

Figure 1: Hydrops fetalis with hypoplastic right lung and extra lobar sequestration

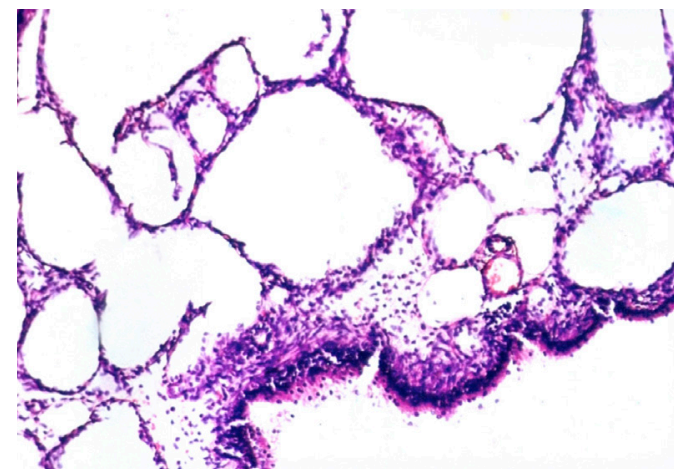

Figure 3A: Back to back arrangement of Bronchiolar-like structures (HE stain, x100).

in approximately $25 \%$ of cases accessioned to the Armed Forces Institute of Pathology. ${ }^{3}$ Conran and Stocker et $\mathrm{al}^{4}$ studied a series of 50 cases of ELS and found associated CCAM in $50 \%$. We present one such case in a 30 year old lady who presented with intrauterine fetal death.

\section{CASE REPORT}

A 30 year old lady G2P1L1 in the 29th week of gestation, presented with pain abdomen, cough with expectoration of 2 weeks and fever with inability to appreciate fetal movements of 2 days. Chest X-ray and laboratory investigations confirmed lobar pneumonia.USG abdomen revealed a single fetus in breech position with features of hydrops fetalis and hypoplastic left lung.. There was no evidence of blood group incompatibility. She delivered a still born, male baby weighing 1900gm with features of hydrops fetalis. An autopsy was performed.

On examination of the thoracic cavity revealed a hypoplastic right lung, large supra-diaphragmatic extra-pulmonary mass attached by a thin stalk to the mediastinal tissue , mediastinal shift to the left. Cut section of both the lung and extrapulmonary mass was homonogenous,pale white with numerous randomly distributed cysts 0.1 to $0.5 \mathrm{~cm}$ in diameter.(fig. 1and 2). The histopathology of the extra pulmonary mass showed a fibrous capsule with underlying

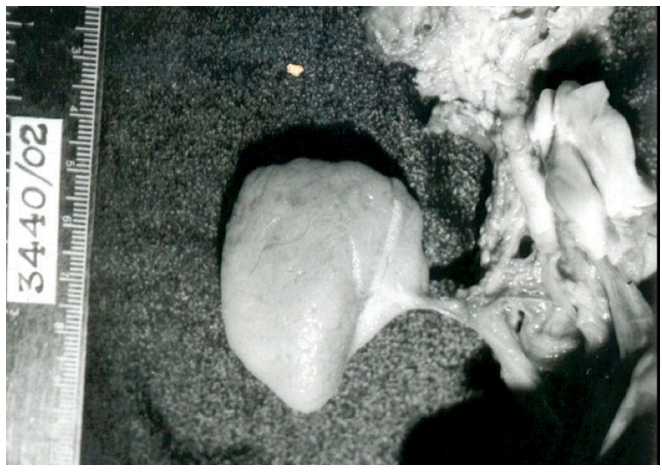

Figure 2: Extralobar sequestration attached with a thin stalk to the mediastinal structures

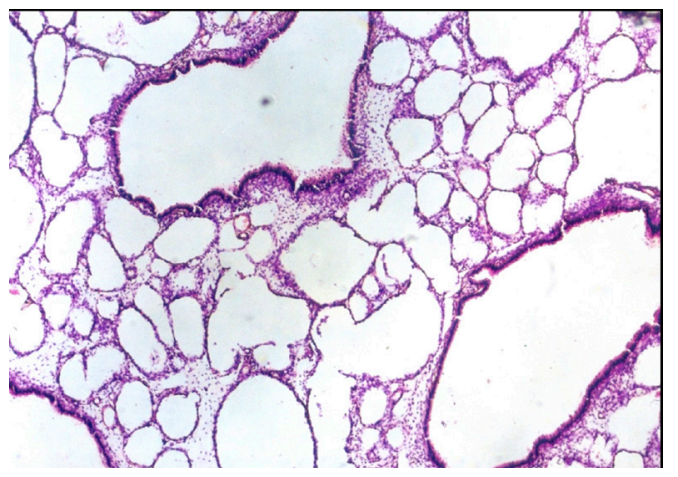

Figure 3B: Cysts lined by cuboidal to columnar epithelium (HE stain, x100).

dilated lymphatics and peripheral lung parenchyma with dilated acini and interspersed bronchioles. The rest of the parenchyma showed multiple cystically dilated spaces and bronchiolar-like structures arranged in a back to back configuration. These structures were lined by cuboidal to columnar epithelium and separated by thin fibrovascular septae. The right lung showed compressed lung parenchyma and similar cysts as described above. The left lung and other viscera were normal. (fig. 3A and 3B)

Based on these features the diagnosis of nonimmune hydrops fetalis with ELS with associated congenital cystic adenomatoid malformation, type -2 and right pulmonary hypoplasia was rendered.

\section{DISCUSSION}

Congenital cystic adenomatoid malformation of the lung is a rare condition occurring in 1 in 25,000-35,000 pregnancies. ${ }^{4}$ At the time of prenatal USG, there may already be evidence of considerable fetal distress, including non-immune hydrops fetalis (NIHF) in these cases (12.43\%).4 Congenital cystic adenomatoid malformation is further subdivided into 5 pathologic types. Type 0 or acinar dysplasia is characterized by firm and small lungs. It is incompatible with life and is seen in association with cardiovascular anomalies and dermal hypoplasia. Type 1 has multiple large cysts or a 
single dominant cyst with surrounding smaller cysts. The lining of the cysts is ciliated columnar to pseudostratified ciliated columnar epithelium. Type 2 is composed of multiple evenly distributed cysts that rarely exceed $1.2 \mathrm{~cm}$ in diameter lined by ciliated cuboidal to columnar epithelium. Type 2 lesion is seen in association with extralobar sequestration in $40 \%$ of the cases. Type 3 lesions present with mediastinal shift and large bulky masses with small cysts. Type 4 CCAM known as the peripheral cyst type is distal acinar in origin. The common clinical presentations of CCAM are stillbirth or neonatal death associated with anasarca, prematurity and maternal polyhyadraminos, acute respiratory distress in the newborn or an indolent course with recurrent pulmonary infections..$^{5-8}$ Congenital cystic adenomatoid malformation can cause considerable morbidity and mortality secondary to pulmonary hypoplasia $(\mathrm{PH})$ because of lung compression and NIHF due to compression of the heart and great vessels. The presence of hydrops has emerged as the single most important poor prognostic indicator in fetuses with congenital cystic adenomatoid malformations. The co-existance of CCAM in ELS has been rarely reported. ${ }^{1,2,5}$ Extralobar sequestration cases containing back- to-back cystic structures of varying sizes lined by bronchiolar type epithelium are classified as CCAM arising in an extralobar sequestration. The age at diagnosis ranges from prenatal period to 73 years. ${ }^{5,6,9}$ There is an equal gender distribution with $74 \%$ being left sided lesions. Whereas ELS can be readily distinguished from intralobar sequestration by the complete separation of ELS from the normal lung, the presence of CCAM within on ELS requires careful microscopic examination to identify the features mentioned above. CCAM in association with ELS is invariably of the type 2 morphology. Thus, ELS, a developmental anomaly of the lung seen in the thoracic spaces, anterior and posterior mediastinum, and within the diaphragm, may contain a second anomaly, CCAM usually type 2 , in upto $50 \%$ cases.

\section{REFERENCES}

1. Halloran G, Silverberg S.G, Salzberg A.M. Congenital cystic adenomatoid malformation of the lung. Arch. Surg 1972;104:715-9. Crossref

2. Oster A.G, Fortune D.W. Congenital cystic adenomatoid malformation of the lung. Am. J. Clin. Pathol.1978;70:595-604. Crossref

3. Stocker JT, Kagan -Hallet K. Extra lobar pulmonary sequestration : analysis of 15 cases. Am J Clin Pathol 1979;74:917-45. Crossref

4. Zangwill BC, Stocker JT. Congenital cystic adenomatoid malformation with in an extralobar pulmonary sequestration. Pediatr Pathol 1993;13:309-15. Crossref

5. Vu L, Tsao K, Lu H, Nobuhara K, Farmer D, Harrisan M, Goldstein RB. Characteristics of congenital cystic adenomatoid malformations associated with non -immune hydrops and outcome. J Pediatr Surg
2007;42:1351-6. Crossref

6. Conran RM, Stocker JT. Extralobar sequestration with frequently associated congenital cytic adenomatoid malformation, Type 2: Report of 50 cases. Peadiatric and Development pathology 1999;2:454-63. Crossref

7. Jain A, Anand K, Singla S, Kumar A Congenital cystic lung diseases. J Clin Imaging Sci. 2013;30;3-5

8. Shanmugam G, MacArthur K, Pollock JC. Congenital lung malformations--antenatal and postnatal evaluation and management. Eur J Cardiothorac Surg. 2005;27:45-52. Crossref

9. Giubergia V, Barrenechea M, Siminovich M, Pena HG, Murtagh P. Congenital cystic adenomatoid malformation: clinical features, pathological concepts and management in 172 cases. J Pediatr (Rio J). 2012;88:143-8. Crossref 